\title{
The Difference on Pain and Range of Motion After High-Intensity Laser Therapy on Myositis Ossificans of the Right Deltoid Muscle: A Case Report
}

\author{
Dian Herdiansyah, Tertianto Prabowo, Rachmat Z. Goesasi
}

Physical Medicine and Rehabilitation Departement, Faculty of Medicine, University of Padjadjaran, Bandung

\begin{abstract}
Background: Myositis Ossificans (MO) is a non-neoplastic proliferation of bone and cartilage tissue after micro-injuries on muscle, as a result of dysregulation response to inflammation on stem cells.

Results: A patient, 50-years old man with pain and restricted movement of the right shoulder, that rose 8 hours after exercise. The radiograph revealed the MO on the right Deltoid muscle. The patient has received the high intensity of laser therapy for two continuous therapy. The result after therapy was decreased of pain and can move the right shoulder easily. Furthermore, after two times/week for four weeks of therapy, there was no pain, as well as the x-ray was showed reduced the size of calcification.

Conclusion, The high intensity of Laser therapy was improved the pain dan reduced the size of MO on right deltoid muscle
\end{abstract}

Keywords: Myositis Ossificans, Deltoid Muscle, High Intensity of Laser Therapy

\section{Correspondence Detail:}

Dian Herdiansyah,MD

E-mail: dd_herdiansyah@yahoo.co.id

\section{INTRODUCTION}

The Myositis ossificans (MO), is a pathologic bone formation that occurs in soft tissues that do not normally ossify. It is a rare condition with unknown pathogenesis ${ }^{(1)}$ It was a self-limiting disease, a benign ossifying lesion that can affect any soft tissue including the subcutaneous fat, tendons, and nerve. However, the most prevalence found in the muscle as a solitary lesion. The cellular mechanism of heterotopic bone formation is the result of the dysregulation of local stem cells in response to tissue injury and subsequent inflammation. (2) 
The High-Intensity Laser Therapy (HILT) was relatively recent, constant evolution technology, and has approved by FDA in 2004. The HILT was a high power pulsed neodymium-doped yttrium aluminum garnet (Nd: YAG). The high peak powers of HILT were able to reach and stimulate organs that are difficult to reach by classical lasers, such as the deep joints. Nowadays the use of pulsed Nd: YAG Laser has been spreading in the therapy for pain management, and have excellent results. Studies exist which describe the anti-inflammatory, anti-inflammation and antalgic effects of Nd: YAG Laser ${ }^{(3)}$

This case report was a woman (50 years) has pain and restricted right shoulder movement, after highintensity weight training for six weeks on the shoulder muscles, has diagnosed with MO on the right deltoid muscle, and have got the HILT.

\section{CASE REPORT}

The fifty years-old women visited at Physical medicine and rehabilitation department with the pain on the right medial deltoid muscle since six weeks ago. The pain intensity by visual analog scale (VAS) was 5 . She was still done some shoulder exercise for two weeks, and after seven weeks of exercise the pain was getting worse, even during the rest period. There was a limitation of shoulder range of motion, like a rising hand and as the consequences, the patient cannot do any activity that requires rising hand motion on the right shoulder. There were no history of recent infection, previous pain intervention, and traumas or motor vehicle accidents on the right shoulder.

The physical examination showed slight swelling at the insertion of the right deltoid muscle with tenderness by palpation. Patient felt pain and limited movement when she moved the shoulder to flexion and abduction. There was a weakness on some right shoulder muscle; flexor and abductor that the score by manual muscle testing was three and four. The plain radiograph on the right shoulder showed calcification at the right deltoid muscle has shown in Figure 1.

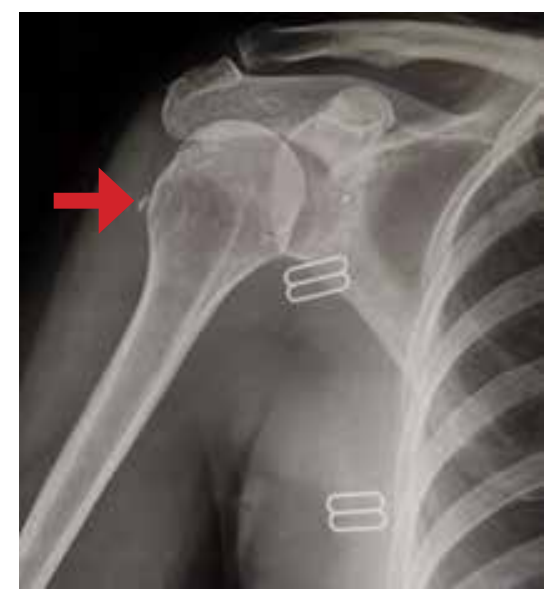

Figure 1. Radiographs were taken six weeks post-trauma demonstrating the appearance of ossification in the insertion of right deltoid muscle. 
The management for this patient was using High-Intensity Laser Therapy (HILT) treatment with pulsed Nd: YAG laser produced by a HIRO 3 device (ASA, Arcugnano, Vicenza, Italy) : with pulsed emission (1,064 $\mathrm{nm}$ ), very high peak powers (3 kW), high levels of fluency (energy density) $(1,780 \mathrm{~mJ} / \mathrm{cm})$, brief duration $(120 \mu \mathrm{s})$, and a duty cycle of approximately $0.1 \%$, This patient was used three times slow scan $(3 \mathrm{~cm} / \mathrm{s})$ method per session with instrument parameters : first scan (intensity $810 \mathrm{~mJ} / \mathrm{cm}^{2}$, frequency $30 \mathrm{~Hz}$ ), second scan (intensity $970 \mathrm{~mJ} / \mathrm{cm}^{2}$, frequency $25 \mathrm{~Hz}$ ), and third scan (intensity $1070 \mathrm{~mJ} / \mathrm{cm}^{2}$, frequency $20 \mathrm{~Hz}$ ) total energy $1250 \mathrm{~J}$ per session, twice a week, for 4 weeks.

After two sessions of therapy, a patient reported the decreased of pain (VAS: 3) and able to move her right shoulder to all direction. After eight sessions (four weeks) followed up, the patient had not felt the pain. Figure 2 has described the Plain Radiology x-ray on the right shoulder that showed the reduced of calcification at the right deltoid muscle.

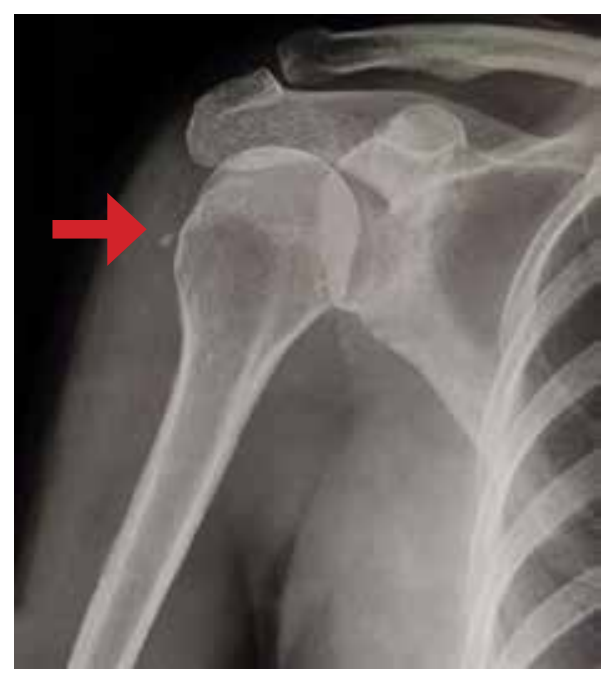

Figure 2. Radiographs forth weeks post HILT therapy is demonstrating the appearance in reducing the size of ossification in the insertion of the right deltoid muscle.

\section{DISCUSSION}

Myositis Ossificans (MO) is a nonneoplastic proliferation of bone and cartilage tissue at the site of a previous injury, most commonly after blunt trauma or repeated micro-injuries. Myositis ossificans is also known as a Myositis ossificans traumatic (MOT) because in over half of the cases exists a direct relationship with a single or repetitive injury of the muscle. ${ }^{(4)}$ Myositis ossificans, in its literal translation, implies an inflammatory ossification of muscle. Kan et al. demonstrated that the cellular mechanism of heterotopic bone formation was the result of the dysregulation of local stem cells in response to tissue injury and subsequent inflammation. ${ }^{(2)}$ The minor trauma, such as repetitive minor mechanical injuries, and inflammation, are believed has an association with the development of myositis ossificans. ${ }^{1-3}$ 
Diagnosis can obtain from the clinical history, physical examination, radiographic imaging, and less commonly by diagnostic ultrasound or magnetic resonance imaging (MRI). Post-traumatic myositis ossificans occurs as a complication in approximately $20 \%$ associated with muscle contusions and strains. It is responsible for considerable morbidity, typically present with localized tenderness, swelling, and decreased the range of motion. ${ }^{4,5}$ In this case, the MO was caused pain accompany by the loss of range of motion, and the mass was found by palpation in the weeks following trauma. Besides, the radiographic signs of ectopic bone usually develop approximately $3-5$ weeks after the injury ${ }^{4}$

The differential diagnosis that should be considering is non-neoplastic disorders or malignant tumors. The most frequent clinical misdiagnoses were osteosarcoma. ${ }^{6}$ Pain and swelling in osteosarcoma are persistent and progressive. It has a periosteal reaction and cortical destruction present on bone radiographs. ${ }^{1}$ Other differential diagnosis that might take into consideration is local infection with pain and swelling accompanied by fever. ${ }^{2}$

The pathogenesis and primary etiological factors of bone formation remain unclear. ${ }^{2,47}$ Inflammation caused by the damage, swelling and tenderness, recruits an influx of inflammatory cells and macrophages into the necrotic tissue that releases osteogenic bone mediators which encourage the formation of heterotopic bone. There is cellular damage causing necrotic debris that subsequently removed through the invasion of inflamed cells. Fibroblasts from the endomysium then assail the injured cells, and mesenchymal cells begin to proliferate. The fibroblasts and mesenchymal cells produced osteoid and chondroid tissue that lays down the groundwork for the formation of bone within the damaged tissue. These mesenchymal cells may differentiate when exposed to an inflammatory rich component. ${ }^{2,7}$ In most cases, there was spontaneous resorption. The smaller lesions in the upper extremity have resorbed completely, while large lesions near the insertion or the origin of the muscle are more persistent. The smaller MO was a self-limiting condition and resolving in about six months., ${ }^{8,9}$

Initial treatment of the acute MO is to reduce local inflammation which is hypothesized to reduce the chance of developing MO. ${ }^{(4)}$ The expression and secretion of the inflammatory cell can inhibit by Laser therapy exposure. ${ }^{(10)}$ Most of all cells increase their metabolism when irradiated with light laser therapy. The effects included an increase in the synthesis of ATP that will improve the acceleration of leukocyte activity leads to faster removal of cellular components and non-viable tissue. As a consequence, the Laser Therapy improves reabsorption of bone mass in $\mathrm{MO}$, as well as improve regeneration and healing processes. ${ }^{11}$

Indeed, the evidence for the effectiveness of the Laser therapy on MO was still limited. 


\section{CONCLUSION}

The pain intensity and the range of motion of the shoulder were an improvement that has achieved after two sessions of the Laser therapy. Reduce the pain accompanied by reducing the size of calcification according to X-Ray examination after eight sessions. It has shown that Laser therapy was the promising treatment modality for the management of the MO. The Laser therapy is an interesting non-invasive, and affordable modality for the treatment of the MO.

\section{REFERENCES}

1. Hendifar Andrew E. JD, Arkfeld Daniel G.. Myositis Ossificans: A Case Report. Arthritis \& Rheumatism 2005; 53: 793-5.

2. Walczak Brian E. D, Johnson Christopher N., DO, Howe B. Matthew, MD. Review Article Myositis Ossificans. J Am Acad Orthop Surg 2015; 23: 612-22.

3. Thabet Ali Abdel Monsif MSM, Ali Mohamed Mohamed Ibrahim, Helal Omar Farouk High-Intensity Laser Versus Low-Intensity Laser Therapy in Management of Postmenopausal Osteoporosis. In: Bull Fac Ph Th Cairo Univ. 2011. p.73-80.

4. Torrance David Allen dC. Treatment of post-traumatic myositis ossificans of the anterior thigh with extracorporeal shock wave therapy. J Can Chiropr Assoc 2011; 55: 240-6.

5. Schultzel Mark M. JMH, Rosenthal Howard G. Bilateral Deltoid Myositis Ossificans in a Weightlifter Using Anabolic Steroids. In: Healiocom/Orthopedics. 2014.

6. Sorin Claudiu Man CNS, Otilia Fufezan, Gheorghe Mihut. Myositis Ossificans Traumatica of the Neck - a Pediatric Case. Maedica - a Journal of Clinical Medicine 2011; 6: 128-31.

7. Muir B. Myositis ossificans traumatic of the deltoid ligament in a 34-year-old recreational ice hockey player with a 15-year post-trauma follow-up: a case report and review of the literature. J Can Chiropr Assoc 2010; 54: 229-42.

8. Saartje Defoort NAA, P.D. Debeer. Myositis ossificans circumscripta of the triceps due to overuse in a female swimmer. International Journal of Shoulder Surgery 2012; 6: 19-22.

9. Dash N. KAS. Myositis ossificans. Medical journal armed forces India 2012; 6: 407-11.

10. Bjordal Jan Magnus L-MRABo, Joensen Jon, Iversen Vegard Vereide. The anti-inflammatory mechanism of low-level laser therapy and its relevance for clinical use in physiotherapy. Physical Therapy Reviews 2010; 15: 286-93.

11. Antonio MME. Laser Therapy in Inflammation: Mechanisms, techniques, and instrumentation (dissertation). Coimbra: University of Coimbra. 2014. 